\title{
La place du nom propre dans les dictionnaires collaboratifs : le cas de La Parlure
}

\author{
Dolar, Kaja \\ Université Paris Ouest-Nanterre La Défense / MoDyCo - Université de Ljubljana \\ dolar.kaja@gmail.com
}

\section{Introduction}

Suite à l'émergence des nouvelles technologies et des possibilités qu'elles offrent, nous assistons à la floraison des dictionnaires dits collaboratifs. A la différence des dictionnaires numériques/informatisés, qui sont en réalité des dictionnaires académiques mis en ligne, la particularité des dictionnaires collaboratifs réside dans le fait que leur rédaction soit proposée au grand public; elle se fait par les internautes, sans intervention de linguistes ou spécialistes de la lexicographie. Avec leur apparition, la lexicographie a sans doute pris un nouveau tournant en mettant en avant une tendance actuelle, la lexicographie participative.

Dans cet article, nous nous intéresserons en premier lieu à la forme particulière de ces dictionnaires ainsi qu'à leur fonctionnement général. A ce propos, nous nous pencherons plus précisément sur le dictionnaire collaboratif français La Parlure pour analyser son fonctionnement ainsi que certaines des particularités qui le caractérisent et le distinguent des autres dictionnaires de ce genre.

Dans un deuxième temps, nous nous interrogerons sur la place du nom propre dans ces dictionnaires collaboratifs. Nous analyserons quelques entrées relevant du nom propre dans le dictionnaire collaboratif La Parlure pour savoir comment le nom propre s'insère dans la nomenclature ainsi que dans l'ensemble $\mathrm{du}$ dictionnaire. Nous nous demanderons également comment le nom propre contribue à la création lexicale des entrées de La Parlure. Enfin nous traiterons de l'émergence de nouvelles unités lexicales formées sur la base de noms propres. Par cela nous montrerons en quoi le nom propre contribue à l'innovation lexicale en français contemporain.

\section{Les dictionnaires collaboratifs}

La lexicographie traditionnelle a été récemment confrontée à l'émergence d'une nouvelle forme de dictionnaires: les dictionnaires numériques collaboratifs que nous appelons également participatifs. Il s'agit - comme le nom l'indique - d'une base de données collectives, ouverte à tout le monde. De ce fait, les dictionnaires collaboratifs présentent souvent un grand nombre d'expressions créatives et de nombreuses manifestations de la langue parlée. Ils illustrent des usages discursifs nouveaux associés à une communauté de locuteurs en même temps que la pratique culturelle qui transparaît dans cette création lexicale.

Ces dictionnaires se distinguent nettement des dictionnaires numériques/informatisés (dictionnaires académiques mis en ligne) comme des donnés numérisées qui peuvent se présenter sous la forme d'index ou de concordances (Eluerd 2000 : 104-106). Ils ont été jusqu'ici peu décrits, analysés et conceptualisés et c'est pourquoi nous étudierons - avant de nous focaliser sur le nom propre et son rôle dans l'innovation linguistique - le fonctionnement des dictionnaires collaboratifs, leur forme particulière ainsi que leurs particularités par rapport aux dictionnaires traditionnels. Nous avons choisi de nous pencher plus en détail sur le dictionnaire collaboratif du français nommé La Parlure. 


\subsection{La forme et le fonctionnement des dictionnaires collaboratifs}

La création de la base lexicale d'anglais Urban Dictionary, le premier dictionnaire collaboratif - datant de 1999 et comptant à l'heure actuelle plus de 7000000 définitions -, a eu un effet boule de neige et aujourd'hui une masse de dictionnaires participatifs plus ou moins spécialisés sont présents sur internet. Dans la conception du projet, le dictionnaire est perçu comme un objet libre et accessible à tous, aussi bien pour la consultation que pour la rédaction. Ainsi les internautes créent et remplissent eux-mêmes les entrées.

Pour cela, deux types d'action leur sont proposées : 1) l'ajout d'une nouvelle entrée lexicale, 2) la modification d'une entrée existante. Dans le dernier cas, le contributeur ne pourra pas effacer la contribution précédente mais pourra ajouter des informations. Ce cas de figure est illustré dans l'exemple suivant qui montre les différentes phases d'éditions successives au cours desquelles les contributeurs viennent amender la première définition proposée.

\section{Habillé comme la chienne à

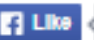 \\ Jacques (Québec)

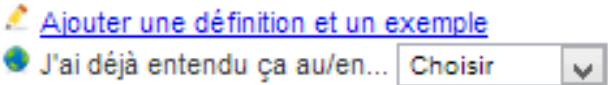

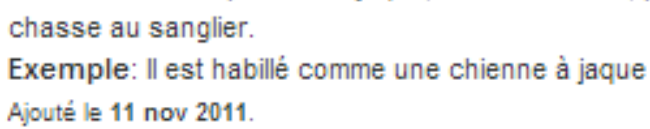

Notons d'abord que l'ordre des entrées va de la plus récente (en haut) à la plus ancienne (en bas). Cela signifie que le premier rédacteur a écrit une définition assez courte (mal habillé). Le suivant y a ajouté une anecdote qui expliquerait l'étymologie de la locution. D'ailleurs, la présence du déictique je inhabituelle pour les gloses définitoires des dictionnaires classiques - s'explique par le fait que celles-ci ne sont pas rédigées par les lexicographes. Le troisième rédacteur s'oppose au deuxième, en précisant que le lexème chienne dans la locution habillé comme la chienne à Jacques doit être pris au sens figuré. Le dernier rédacteur (qui figure tout en haut) propose une définition complètement différente (vêtement 
court). Cet exemple complexe met en évidence à la fois les différentes phases successives de la rédaction et aussi un certain flottement sémantique.

Du fait du caractère collaboratif du dictionnaire, chacune des entrées peut donner lieu, à tout moment, à un ajout ou un commentaire - émanant du rédacteur ou d'un autre contributeur - et ainsi être considérée comme une « conversation chroniquement en cours » selon le terme de Goffman (1987). Cette relation en constante évolution entre le texte existant et les éventuels ajouts ou réponses qui pourraient être produits rend les entrées des dictionnaires collaboratifs constitutivement dialogiques.

Les entrées proposées là sont généralement d'origines très diverses. Beaucoup sont marquées par la présence de formes issues de l'oral, d'expressions du quotidien, de mots étrangers ou de slogans publicitaires. Certaines sont créées à partir des matrices dites externes (emprunts). Selon les entrées et les dictionnaires, la structure de l'article se rapproche plus ou moins d'une entrée dictionnairique classique : l'entrée est parfois suivie de sa catégorie grammaticale (nom, adjectif, verbe) et/ou de son origine et/ou de ses particularités morphosyntaxiques. Quelques dictionnaires proposent également des synonymes exacts ou approximatifs. Les gloses définitoires sont aussi fréquemment accompagnées de plusieurs exemples d'emplois venant illustrer l'usage défini et renvoyant même parfois à un lien vers un réseau social sur lequel le terme a été employé. Il faut également signaler que, les contributions n'étant pas signées, les rédacteurs restent anonymes, ce qui rend centrale la question de la légitimité et de l'attestabilité des gloses proposées. Les liens vers des citations sur des réseaux sociaux ou autres sites et l'utilisation fréquente d'exemples sous la forme de discours directs guillemetés semblent précisément jouer un rôle dans la légitimation des définitions proposées.

Etant donné que l'attestabilité des entrées repose uniquement sur les propositions des contributeurs certaines entrées pouvant être des hapax ou relever plutôt d'une créativité lexicale personnelle que d'une expression largement utilisée - les dictionnaires collaboratifs ne peuvent être considérés comme des corpus représentatifs mais doivent avant tout être compris comme un phénomène social qu'il faut traiter comme tel. Un dictionnaire collaboratif présente les usages d'une communauté de locuteurs et il est en conséquence riche en formes émergentes et pratiques discursives nouvelles. Ces dictionnaires semblent donc constituer un objet d'étude fécond pour l'observation de l'innovation lexicale, les entrées décrivant des usages pour la plupart non-standards (emprunts, sens détournés, expressions d'argot ou vulgaires etc.). Ils recensent des expressions que les locuteurs emploient au quotidien, et c'est en quoi ils représentent selon nous un observatoire des usages en circulation. Pour toutes ces raisons, nous considérons donc les dictionnaires collaboratifs comme un bon corpus des pratiques discursives, révélateur de pratiques langagières émergentes.

\subsection{Le dictionnaire collaboratif du français : La Parlure}

Après avoir caractérisé le fonctionnement général des dictionnaires collaboratifs, nous allons maintenant étudier plus en détail un spécimen particulier : La Parlure. Il s'agit d'un dictionnaire collaboratif du français qui provient du Québec. Il faut souligner que La Parlure n'est pas a priori un dictionnaire des usages québécois. Néanmoins nous en constatons une présence importante, car de nombreuses formes s'expliquent par la coexistence du français et de l'anglais : certaines entrées, notamment les calques en français québécois, résultent de la situation de contact linguistique (Mérillou 2003 : 401-405). A ce propos, notons que l'innovation linguistique survient dans les situations où le français est parlé aussi bien en langue première qu'en langue seconde ou étrangère (Thauvin-Chapot $2003: 433$ ).

Pour autant, La Parlure (accessible sur le site : www.laparlure.com) est très loin de se limiter au français québécois, l'objectif étant de créer une base des expressions francophones. Le site, dont les débuts remontent à 2009, est édité par Greg Sadetsky et l'ensemble du projet est associé à une petite maison d'édition montréalaise (Moult Éditions). La nomenclature du dictionnaire compte à l'heure actuelle plus de 3700 entrées (1059 définitions en 2009, 1168 en 2010, 1185 en 2011 et 689 en 2012).

Pourquoi La Parlure? Il existe au moins deux dictionnaires collaboratifs du français, lesquels se distinguent tant par leur contenu que par leur fonctionnement. La Parlure, qui nous servira de corpus, est 
entièrement collaboratif et complètement libre. L'administrateur doit seulement gérer la base de données en veillant aux discours de haine et en effectuant des actions de mise en page minimale. En revanche, le Dictionnaire de la Zone, hébergé sur le site : www.dictionnairedelazone.fr, fonctionne différemment. Les internautes peuvent soumettre des propositions qui sont ensuite revues par l'administrateur. Celui-ci décide de mettre en ligne ou non les entrées. Outre la mise en forme, il peut également compléter la définition et les exemples. Dans l'ensemble, son intervention ressemble plus au travail d'un éditeur et le site est plus proche du fonctionnement d'un blog. Le contenu est également différent : le Dictionnaire de la Zone se restreint à l'argot de banlieue, c'est-à-dire au parler des cités. Les différences entre les deux dictionnaires collaboratifs français s'expliquent donc aussi bien par le contenu que par les objectifs des deux projets. Dans le cadre de notre recherche, nous nous limiterons à l'étude de La Parlure.

Par rapport au fonctionnement général des dictionnaires collaboratifs, on peut mettre en avant plusieurs particularités de La Parlure, qui propose - outre la création de nouvelles entrées - l'ajout d'une définition et d'exemples aux entrées déjà existantes. Par contre, le rédacteur ne pourra pas supprimer ce qui a déjà été saisi : les définitions et les exemples existants ne peuvent être modifiés. Les différentes gloses définitoires sont ainsi données à la suite (sans pour autant être numérotées), allant de la plus récente à la plus ancienne : d'où une mise en page assez singulière. Notons également que la date de la création des entrées figure systématiquement. Le rédacteur peut en outre bénéficier de la publication de l'entrée sur le réseau social Facebook, ce qui contribue à la popularisation du dictionnaire.

Par ailleurs, le dictionnaire met en avant la francophonie en insistant sur la variation dite géographique, qui rend compte des différents usages en fonction des lieux (Gadet 2007) : le site propose aux rédacteurs ainsi qu'aux lecteurs de «situer» l'expression. Ainsi, ils peuvent choisir «j'ai déjà entendu cette expression en/au Québec/Canada/Belgique/France/Autre Pays ». Puis, un deuxième menu descend pour choisir la région et la ville. Ils peuvent également évaluer les définitions en cliquant sur l'icône voisine. Ils votent ainsi pour ou contre la définition (de la même façon que les lecteurs peuvent évaluer les commentaires sur les blogs, par exemple).

Pour ce qui concerne son fonctionnement, La Parlure semble donc interactive. Quant à sa mise en forme, elle paraît quelque peu rudimentaire, et l'éditeur en est conscient. Pour accéder aux entrées, le site propose de passer par les pages et non pas en suivant ordre alphabétique (ce qui reste un projet à venir). Une carte des expressions selon l'endroit où elles ont été notées ou entendues, la correction collaborative par les utilisateurs et la liste des expressions les plus populaires sont également prévues.

\section{Le nom propre dans des dictionnaires collaboratifs}

Le nom propre, en tant que tel, représente un phénomène linguistique à part entière et suscite de nombreux problèmes méthodologiques. Son étude est longtemps restée exclue de la linguistique, étant un sujet de la philosophie (logique) et de la philologie. La bascule des analyses vers le champ linguistique a pour conséquence une multitude de définitions différentes du nom propre en fonction du cadre théorique retenu. Pour notre étude, nous laisserons de côté les critères classiques (tels que la majuscule initiale, le caractère intraduisible du nom propre, etc.) qui peuvent être largement discutés, pour nous fonder sur la définition suivante, qui met en avant le rôle que joue l'énonciation dans l'analyse, l'institution et l'interprétation des noms propres :

La définition du nom propre comme signe linguistique contient, outre le signifiant et le signifié, le référent, c'est-à-dire un renvoi à l'actualisation du signe dans le discours (...); d'autre part, le sens d'un nom propre - "x appelé n par s" - est défini comme une relation de dénomination entre un individu (x) et un nom propre (n) exprimée par un sujet énonciateur (s), qui dépend d'un acte de nomination associant tel nom à tel individu et initiant ainsi l'usage du nom. La valeur des variables $\mathrm{x}, \mathrm{n}$ et $\mathrm{s}$ est déterminée pour une occurrence de nom propre donnée, en fonction de la situation d'énonciation et d'un acte de nomination logiquement préalable. La notion linguistique de personne permet précisément de rendre compte de ce rapport entre 
référent du nom propre et énonciation et du rôle du contexte dans l'interprétation du nom propre.

(Cormier $2011: 5,2013:$ 173-174)

La catégorie du nom propre est loin d'être homogène ; on y trouve des unités sémiotiques extrêmement diverses. Pour notre analyse, nous proposons une typologie en reprenant les termes de Vaxelaire, qui se donne pour but de catégoriser les différents noms propres en fonction de la nature de leur référent. Nous retrouvons principalement deux catégories récurrentes : premièrement, les anthroponymes, qui désignent des personnes - les noms de famille, prénoms, surnoms, pseudonymes, sigles etc. Deuxièmement, les toponymes, qui désignent des lieux - unités administratives (pays, régions, provinces, villes, départements), unités géographiques (continents, îles, péninsules, mers, océans, lacs, fleuves, rivières, canaux, montagnes, déserts, forêts, vallées, grottes etc.), astres, rues, autoroutes, gares, cimetières, places, adresses, etc. Au demeurant, Vaxelaire s'appuie sur la sémantique interprétative de Rastier pour introduire une catégorisation beaucoup plus fine, en tenant compte également des noms propres des peuples, directions géographiques, manifestations naturelles, animaux et objets anthropomorphes, noms commerciaux, noms de regroupements humains, produits de l'activité humaine, médias, titres d'œuvres, divertissements, calendriers, événements, nouvelles technologies, fiction, etc. (Vaxelaire 2005 : 312-398).

Le passage du nom propre au nom commun est parfois complexe. Afin de contourner ce problème, certains chercheurs proposent la notion des noms propres modifiés : les noms propres perdent «leur caractère 'unique' et 'singulier' »(Kleiber 1981: 332). Nous trouvons ainsi les différents emplois déviants du nom propre tels que l'emploi de fractionnement, dénominatif, exemplaire, métaphorique et métonymique (Vaxelaire 2005 : 301). Du reste, le nom métaphorique s'est avéré extrêmement présent dans La Parlure. Comme son nom l'indique, il se caractérise par sa valeur figurée :

La métaphorisation du Npr consisterait donc en un rapprochement entre deux domaines encyclopédiques distincts. A l'intérieur d'un de ces domaines le référent original du Npr occupe une position privilégiée, qui l'a rendu plus ou moins célèbre. Lorsque cette structure ou figuration est appliquée à un des domaines encyclopédiques dans lesquels s'inscrit le référent topique, il s'avère que la position de celui-ci dans ce nouveau domaine peut être comparée à celle du référent original dans le domaine initial.

(Jonasson $1991:$ 79)

Le nom propre est donc « interprété comme dénotant un rôle qui peut être assumé par plusieurs individus différents » (Jonasson 1994 : 174). Par ailleurs, cette notion se rapproche fortement du nom propre désincarné se rapportant aux «formes lexicales et phoniques, qui ont d'autres fonctions que la référence et qui n'ont de lien durable avec aucun particulier» (Gardiner 1954 dans Jonasson 1994: 175). L'interprétation du nom métaphorique se fait grâce au lien d'analogie entre le référent désigné par le nom propre et le référent réel de la communication; dans le cas des anthroponymes, « on a une classe réelle dont on sélectionne un (ou plusieurs) individu(s), distinct(s) du référent du nom propre, qui ne fait pas luimême partie de la classe » (Gary-Prieur 1994: 145). Cette analogie s'établit au moment de l'interprétation et en fonction de la situation de communication. Les noms métaphoriques peuvent garder seulement «certaines propriétés » des noms propres (Gary-Prieur 1994: 47). Nous y reviendrons à propos de l'étude des cas.

Mis à part les modifications référentielles, où le référent du nom propre change, il faut également tenir compte des modifications formelles, de nature morphosyntaxique, que peut subir le nom propre. Jonasson étudie les structures syntaxiques du nom propre et les classe en 11 catégories, en fonction de(s) élément(s) qui s'ajoute(nt) au nom propre (adjectif pré- ou postposé, déterminant, nom commun). Du point de vue syntaxique, le nom propre peut jouer différents rôles (attribut, épithète, etc.). A part les fonctions référentielle, vocative et la fonction de nomination, Jonasson évoque également les différentes fonctions cognitives et communicatives (Jonasson 1994: 7, 63-112) ce qui montre la complexité de la matière linguistique et les différents problèmes méthodologiques que soulève l'étude du nom propre. Dans cet article, nous allons le traiter uniquement sous un aspect appliqué, à savoir dans son rapport à la lexicologie et la lexicographie. 
En effet, le traitement du nom propre dans les dictionnaires est une des questions centrales de la lexicographie. Niklas-Salminen propose la typologie des dictionnaires suivante : dictionnaires bilingues et monolingues, dictionnaires intensifs et extensifs, dictionnaires des choses et dictionnaires des mots, les premiers correspondant aux dictionnaires encyclopédiques, les deuxièmes aux dictionnaires de langue (Niklas-Salminen 1997: 95). Le nom propre occupe alors une place particulière : il est au cœur des dictionnaires encyclopédiques mais peut aussi se retrouver dans les dictionnaires de langue.

En ce qui concerne les noms propres dans les dictionnaires des langues, Mufwene distingue trois cas différents : l'absence totale des noms propres, noms propres en supplément à la fin et noms propres insérés directement dans la nomenclature (dans ce cas, accompagnés de renseignements purement linguistiques) (Mufwene 1988 : 269). Dans la plupart des dictionnaires classiques d'aujourd'hui, les noms propres sont physiquement séparés du reste de la nomenclature, soit dans une partie distincte du même volume (comme Larousse) soit dans un volume à part (comme Le Petit Robert), même si cette distinction n'a pas été toujours aussi évidente. Pour Quemada, celle-ci est en vigueur depuis le $16^{\mathrm{e}}$ siècle (Quemada 1967 : 311), mais selon Vaxelaire, avant 1700 deux tiers des dictionnaires traitaient des noms propres, seulement un tiers le firent par la suite. Eu égard à la floraison des dictionnaires encyclopédiques, la plupart des dictionnaires de langue après 1789 sont dépourvus de noms propres, même si avant 1700 on n'accordait que peu d'importance à la différence entre le nom propre et le nom commun (Vaxelaire 2005 : 41-43).

Certains linguistes soutiennent que les noms propres ne devraient point figurer dans les dictionnaires de langue mais uniquement dans les dictionnaires encyclopédiques. Ce point de vue est largement contestable, d'autant que les dictionnaires de langue - comme le note Vaxelaire - contiennent de nombreux éléments de type encyclopédique, même s'il est vrai que les entrées traitant du nom propre ne comportent que peu de remarques linguistiques (comme par exemple la prononciation, le genre et la syntaxe des noms propres). Notons aussi la quasi-absence des divers syntagmes qui comprennent des noms propres aussi bien en entrées qu'en sous-entrées (Vaxelaire 2005: 52). Un autre problème important est la place et le traitement d'emplois métaphoriques des noms propres. Ces cas sont rarement traités dans les dictionnaires de langue, même s'ils sont très présents en usage.

Dans la suite de cet article nous allons nous intéresser à la place du nom propre dans le dictionnaire collaboratif La Parlure. Dans un premier temps, nous étudierons les occurrences du nom propre dans la nomenclature pour exposer les différents cas. Puis nous nous intéresserons à la façon dont le nom propre contribue à l'innovation lexicale aussi bien au niveau de sa forme morphosyntaxique qu'au niveau de la construction du sens. Cette étude nous permettra de conclure sur l'apport du nom propre à la créativité linguistique dans le dictionnaire La Parlure.

\subsection{Le nom propre dans La Parlure}

Le dictionnaire collaboratif La Parlure contient 3700 entrées, dont plus de 150 réfèrent directement à un nom propre. Le nom propre peut figurer dans l'entrée même, c'est-à-dire dans le mot vedette (Kodak), dans la définition, c'est-à-dire dans la glose définitoire (savate - Réglisse pour Myriam - Hey ça existe pas Savate, c'est réglisse le vrai mot), dans les exemples (schtrater - Schtrater en Tunisie, zbiber au Maroc, sécher en France - Cet après-midi, je zbibe le cours de Français de "Mazmazelle Izabelle") ou dans les deux (Yasser sti - cri que Jacques Payot lance au stade pour encourager une équipe - Jocelyne Gagné de Val D'or agita ses pompons et Jacques Payot scanda «Yasser sti!» La belle époque quoi.). ${ }^{1}$

Précisons que dans cette étude nous tenons uniquement compte des cas où le nom propre figure dans l'entrée même, c'est-à-dire dans le mot-vedette et non pas dans la glose définitionnelle. Les occurrences où le nom propre apparaît dans les exemples - bien qu'elles soient évidemment très nombreuses - sont également exclues, car elles ne présentent que peu d'intérêt pour notre étude qui se concentre sur la place du nom propre dans l'ensemble du dictionnaire et sur son rôle dans l'innovation lexicale. Malgré la présence constante des noms propres dans les exemples d'usage, nous n'en tiendrons donc pas compte. Il s'agit d'ailleurs d'un trait assez récurrent dans les dictionnaires mais aussi dans les grammaires traditionnelles : alors que les noms propres ne sont pas traités comme entrées du dictionnaire ou comme 
paragraphe spécifique dans les grammaires, ils apparaissent (relativement) abondamment dans les exemples.

Si nous commençons par quelques remarques générales, il faut noter dans un premier temps que les noms propres dans La Parlure sont insérés directement dans la nomenclature, ce qui veut dire que le dictionnaire ne leur réserve pas une partie spécifique, comme c'est le cas, par exemple, du dictionnaire classique Le Petit Robert. Noms propres et noms communs donc sont mêlés dans l'ensemble du dictionnaire. Une deuxième remarque porte sur la forme graphique : bien évidemment, la majuscule initiale seule ne suffit pas à caractériser le nom propre, qui peut être orthographié avec une minuscule initiale (Vaxelaire 2005: 73), mais nous trouvons dans La Parlure des cas déviants dans les deux catégories, aussi bien pour les noms communs que pour les noms propres : les deux sont tantôt notés avec une majuscule tantôt avec une minuscule. Cela s'explique par la nature du dictionnaire, qui est un site entièrement libre, accessible à tous, sans censure ni relecture orthographique. Que les normes orthographique et grammaticale se trouvent souvent écartées, cela ne change pas l'intérêt de l'objet en lui-même, car il ne s'agit en aucun cas d'un dictionnaire normatif ou prescriptif. Même si la problématique de l'orthographe des néologismes est très pertinente (Honvault 2003: 38) nous considérons ce dictionnaire - comme nous l'avons signalé plus haut - avant tout comme un observatoire des usages. C'est pour cette raison que nous n'interviendrons plus sur la forme orthographique dans la suite de l'article : nous ne ferons ni commentaires ni corrections car nous ne souhaitons pas modifier les données du corpus.

Le nom propre en tant que tel apparaît dans la nomenclature de La Parlure sous trois aspects différents :

1) L'entrée porte sur un nom propre standard;

2) L'entrée porte sur un nom propre et un nom métaphorique ;

3) L'entrée porte sur un nom propre et un nom commun.

La catégorie des noms propres standard ou référentiels (Leroy 2004 : 68), c'est-à-dire des noms propres à proprement parler, ne compte pas un très grand nombre d'entrées, par contre elles sont assez diversifiées. Nous y trouvons surtout des toponymes mais ceux-ci se trouvent souvent modifiés morphographiquement (d'où l'intérêt de les traiter dans le dictionnaire) comme c'est le cas de l'exemple suivant :

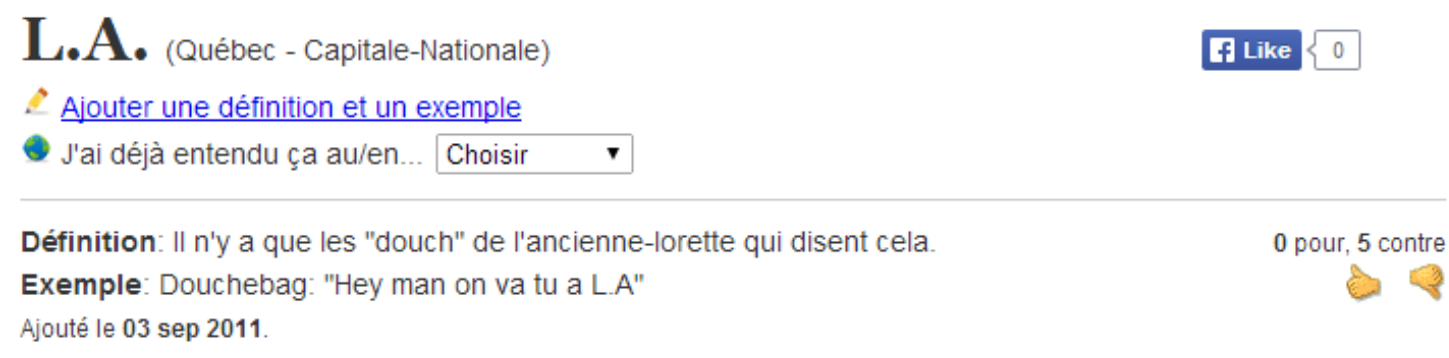

Définition: L'Ancienne-Lorette. Prononcée à l'anglais -- "L.A." comme Los Angeles..

La première glose définitoire (en bas) explique que le nom propre $L$. A. se réfère à l'Ancienne-Lorette malgré l'association directe qui peut se faire en lien avec Los Angeles. La deuxième définition (en haut) est en réalité plutôt un commentaire sur l'usage. Même s'il s'agit d'un nom propre standard, le nom en lui-même subit déjà une modification morpho-graphique (la siglaison), comme c'est également le cas de Hralma - Prononciation du nom de la ville d'Alma (Lac St-Jean) par les Almatois - Heille Steeve, quand 
est-ce tu 'iens dérheuner à Rhalma? où il s'agit d'une écriture phonétique approximative pour imiter la réalisation sonore.

Il y a également des noms propres de personnages et de créatures divines/légendaires, tels que Bonhomme sept heure - Espèce de monstre populaire qui fait mal aux enfants. Vient de "Bone Setter" placeur d'os, ancienne version des chirospraticiens. - T'es mieux d'aller te coucher avant que le bonhomme sept heure passe. ou Sasquatch - Grosse bête mythique et poilue vivant dans les montagnes. Parenté avec le singe. Ta mère est un sasquatch. T'as un sasquatch entre les 2 jambes (s'applique aussi pour la mère). Ici, la glose traite le mot comme un nom propre, tandis que les exemples semblent plutôt le traiter comme un nom commun. En effet, les entrées qui traitent de la valeur standard du nom propre aussi bien dans la glose définitoire que dans les exemples d'usages sont extrêmement rares dans La Parlure.

D'autre part, on constate que le nom propre peut très facilement glisser vers un sens métaphorique, comme dans l'exemple suivant :

\section{Séraphin (Québec)}

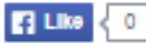

Ajouter une définition et un exemple

J'ai déjà entendu ça au/en... Choisir

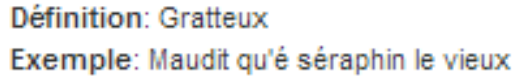

Définition: être un Séraphin - Expression très populaire au Québec tiré du personnage principal d'un téléroman diffusé longtemps à Radio-Canada; Les belles histoires des pays d'en haut. Séraphin Poudrier, maire, usurier notoire était un avare de la pire sorte.

Exemple: "Donne lui 20 S enwoye, fais pas ton Séraphin!"

Ajouté le 10 aoû 2010

La forme du nom propre en elle-même n'a pas subi de modifications morphosyntaxiques ni graphiques ; mais son référent a changé. Cette entrée est particulièrement intéressante, en ce qu'elle explicite les deux aspects séparément, par deux gloses définitoires distinctes. La première (en bas) relève d'un nom propre et la deuxième (en haut) d'un nom métaphorique qui fonctionne comme adjectif. Tout comme dans l'exemple précédant, la glose définitoire et l'exemple d'usage mettent en avant deux valeurs différentes du nom propre, l'exemple se référant à son tour aussi à l'emploi métaphorique et non pas au nom propre proposé dans la glose. Cela peut s'expliquer par le fait que le dictionnaire n'est pas rédigé par des spécialistes mais par les internautes.

Le passage du nom propre au nom métaphorique n'est cependant pas toujours explicité. Alors, un lien métaphorique se crée forcément et l'interprétation se fait par le biais d'une analogie. Citons quelques exemples : marcel leboeuf - qui fait la job mais sans plus - j'ai réparé mon auto c'est Marcel Leboeuf où l'auteur établit clairement une attitude dépréciative vis-à-vis de l'acteur québécois ; Seigneur des anneaux - Adepte de la sodomie forcée. - Louis-Gabriel est un seigneur des anneaux, un pur et dur, il n'y a aucune cenne pour lui résister. Hier encore, il ridait le voisin dans le brun... où la construction du sens joue sur le signifiant du titre de l'ouvrage. L'exemple Durex - Ruban transparent - As-tu du durex? propose un double passage, sauf que ce passage demeure implicite : le nom propre de la marque Durex est remplacé par les produits (préservatifs) qui sont à leur tour des noms communs. La ressemblance formelle permet ensuite d'interpréter le lexème. Notons également que le lien entre le nom propre et le nom métaphorique n'a parfois rien d'évident ni de motivé, comme dans Pepsi - Désignation des Québécois 
par certains Italiens vivant au Québec. - Regarde-moi tous ces Pepsis... L'utilisation de ce type d'expression se fera entre pairs et l'interprétation dépendra du contexte extralinguistique.

Si les noms de marques, tels Dollorama qui propose une adaptation graphique de Dollarama, la fameuse chaine de magasins dont la plupart de la marchandise se situe a 1 dollar ou legerment plus. - J'men va au Dollorama pour m'acheter des sacs de vidange, sont, de façon générale, des noms propres, tel n'est pas le cas pour les noms des produits (Panel - Véhicule de type Ford Econoline. - Tu peux mettre ça dans le panel.) qui sont en réalité des noms communs et non pas des noms propres (Vaxelaire 2005 : 348-350). Nous rencontrons donc ici un problème méthodologique, car le nom du produit (nom commun) remplace fréquemment le nom de la marque (nom propre) :

\section{Kodak (Canada - Ontario)}

\section{Définition: Déf: Caméra}

Du nom d'une marque américaine de caméra. Est passé dans le language populaire comme étant la caméra elle-même.

Exemple: - Passe-moé donc ton kodak, j'ai oublié le mien.

Ajouté le 05 avr 2012.

Non seulement le nom de la marque passe au nom du produit, mais il passe aussi au nom commun désignant l'appareil en général, sans tenir compte de la marque, ce qui illustre bien le passage très courant des noms propres aux noms communs. Ce glissement du mono-référentiel vers le multi-référentiel est certes explicité, mais - contrairement aux exemples précédents - les deux aspects sont réunis dans la même glose définitoire.

Cependant, nous trouvons de rares exemples de passages inverses, c'est-à-dire des expressions imagées pour désigner des lieux réels. Dans ces cas-là, le nom propre se trouve au cœur de la création lexicale. Le syntagme Pisseville composé de pisse (uriner) et ville désigne les toilettes; la procédure d'extension de sens en fait un nom propre (Sablayrolles $2000: 227)$ :

\section{Allez à Pisseville (Québec - Capitale-Nationale)}

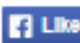

Ajouter une définition et un exemple

J'ai déjà entendu ça au/en... Choisir

Définition: Nom propre. Nom donné ironiquement par les Drummondvillois à

Drummondville en raison de sa situation particulière, à mi-chemin entre Montréal et

Québec.

Exemple: $Y$ a-tu quequ'un qui a besoin d'arrêter à Pisseville?

Ajouté le 13 fév 2011.

Définition: Allez au toillette

Exemple: Faut que j'aille faire un tour à Pisseville

Ajouté le 31 jul 2010 
Nous pouvons conclure cette partie en quatre points : premièrement, le nom propre dans les motsvedettes ${ }^{2}$ est un phénomène plutôt restreint dans le dictionnaire collaboratif La Parlure, ce qui n'est pas très surprenant. Deuxièmement, il peut subir des modifications graphiques (Dollorama) ou morphographiques (L.A.). Troisièmement, le nom propre standard étant quasi absent, il s'associe fréquemment à l'emploi métaphorique. Les noms métaphoriques, qui sont d'ailleurs rarement traités dans les dictionnaires classiques, représentent le cas majoritaire dans La Parlure. Le lien peut être soit implicite (Durex) soit explicité dans les gloses définitoires (Séraphin). Enfin, le passage du nom propre au nom commun est un phénomène bien présent dans La Parlure (Kodak).

\title{
3.2 Nom propre et innovation lexicale
}

Pour l'instant nous nous sommes intéressés aux noms propres figurant tels quels dans la nomenclature du dictionnaire; en même temps, nous avons traité brièvement des noms métaphoriques et des noms communs. Dans tous les cas, la forme initiale du nom propre n'a pas été manipulée dans l'entrée du dictionnaire. Nous verrons maintenant quelles modifications le nom propre peut subir, comment il structure le contenu même des entrées de La Parlure et quel est son rôle dans l'innovation lexicale. Tenant compte de cet aspect, nous pouvons rendre compte de deux phénomènes différents :

1) Le nom propre s'insère dans une locution;

2) Le nom propre subit des modifications morphosyntaxiques.

Arrêterons-nous d'abord brièvement sur la première catégorie, les noms propres dans les locutions que nous définissons en tant qu'ensembles de syntagmes et énoncés minimaux se caractérisant par un transfert sémantique (Rey 2002 : 182-184). Contrairement aux dictionnaires classiques, où nous avons remarqué l'absence de syntagmes qui comprennent des noms propres, cette catégorie est de loin la plus fréquente dans La Parlure: nous trouvons des phénomènes assez diversifiés aussi bien au niveau de la structure grammaticale qu'au niveau du contenu. En ce qui concerne ce dernier, il s'agit en général de valeurs métaphoriques non-lexicalisées semblables aux exemples étudiés plus haut. Ainsi nous trouvons des exemples du type :

\section{Frapper son waterloo (Québec)}

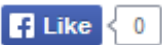

\section{Ajouter une définition et un exemple}

J'ai déjà entendu ça au/en... Choisir

\author{
Définition: Renconter un obstacle infranchissable \\ Exemple: Le CH a renconter son Waterloo devant Boston! \\ Ajouté le 23 sep 2012
}

L'interprétation passe évidemment par les connaissances préalables et les idées associées au terme Waterloo. Les locutions métaphoriques désignant les toponymes sont d'ailleurs très courantes, comme dans Faire la rue Michel - Compléter, terminer une action en cours. Viens du dépôt de cochers rue SaintMichel à Paris à qui les client de la dernière course offraient la course vers le dépôt. - Avec mon pain, si je trouve une tranche de Jambon, ça fera la rue michel! ou alors dans des structures plus complexes telles que Se prendre pour Gibraltar - Se croire invincible, se croire à l'abri du danger Prends-toi pas pour Gibraltar mon p'tit garçon, mets toi un casque pour faire de la motoneige. où un toponyme fait partie d'une construction imagée. Cependant, nous trouvons dans la même catégorie aussi des anthroponymes du type As tu vu la Megan fox? - Avoir repéré une très jolie fille. - Hey vieux, check 
la Megan fox là bas ! Ta raison elle est vraiment belle ! Dans tous ces cas, les noms propres participent «à la construction des énoncés lexicographiques » (Vaxelaire $2005: 49$ ).

Du point de vue grammatical, les noms propres dans les locutions subissent des changements, notamment au niveau du déterminant :

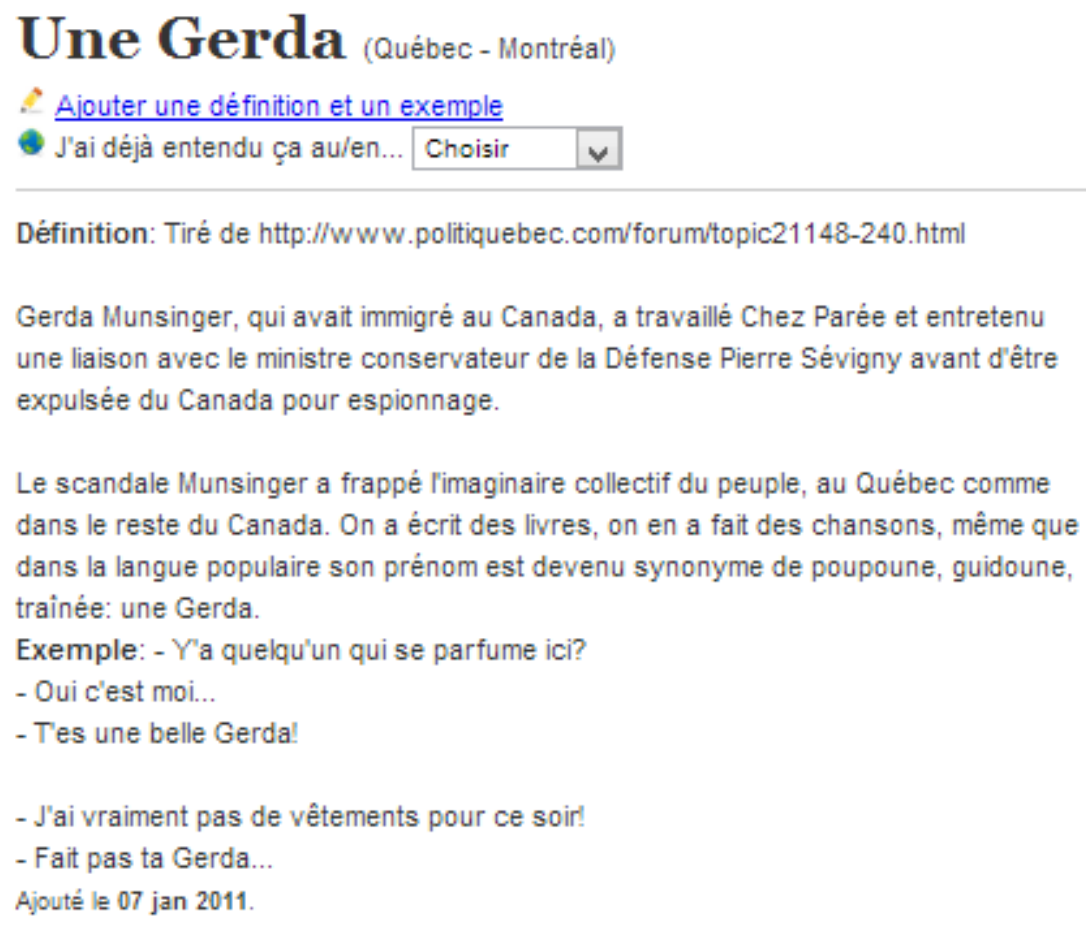

Dans l'exemple cité, le nom propre est précédé de l'article indéfini, ce qui implique un changement de référence (du mono-référentiel au multi-référentiel). La locution T'es une belle Gerda! ou Fais pas ta Gerda ... s'interprète forcément en bloc et en fonction du contexte énonciatif. La valeur du nom propre est ici de nouveau métaphorique. Schnedecker remarque que « dans les situations multi-référentielles, le $\mathrm{Np}$ servirait à marquer l'alternance des référents en position de saillance » (Schnedecker 1997 : 81). De plus, Jonasson puis Gary-Prieur notent que «l'interprétation métaphorique de un $N p$ est beaucoup plus fréquente et beaucoup plus vivante dans une structure où le nom est accompagné d'une expansion » comme un adjectif, une relative ou un complément prépositionnel (Gray-Prieur 1994 : 151). Par ailleurs, le rédacteur indique la source pour renforcer l'attestabilité du lexème en question.

Les noms propres peuvent donc figurer dans les locutions, mais ils peuvent aussi prendre part à des processus morphosyntaxiques. Nous avons déjà mentionné plus haut quelques exemples qui se situent sans doute dans la deuxième catégorie : la siglaison semble être un procédé courant $(L$. $A$. ou encore MLSK ou M-LS-K ou MK - quartier de rue de Pessamit MaLeK-MeSseK - jvien de MLSK PST). La conversion, à son tour, se fait fréquemment du nom à l'adjectif. Les autres cas sont plus rares; dans l'exemple Môsusse (pour Mö̈se), outre l'adaptation graphique, nous pouvons observer la recatégorisation du nom propre en interjection.

Dans la même rubrique, nous pouvons mentionner les emprunts partiellement ou entièrement adaptés, comme être le King de la Wing qui provient du nom du jeu King of fighters Wing. Dans le même sens va également l'exemple suivant: 


\section{Twitter ${ }_{\text {(France) }}$}

\author{
- Ajouter une définition et un exemple \\ J'ai déjà entendu ça au/en... Choisir v \\ Définition: Faire le twit en utilisant seulement Twitter. \\ Exemple: Y'twitte en masse.
}

Ajouté le 27 mai 2009

\section{f Like $\{0$}

4 pour, 5 contre

Twitter est le nom propre du réseau social, mais il est ici défini comme verbe. Ce cas est très intéressant car il rend compte de la création verbale et le fonctionnement des matrices dites externes (Pruvost et Sablayrolles 2003 : 117). En effet le verbe anglais signifiant faire des tweets (commentaires) sur le réseau Twitter est tweet. C'est à partir de ce verbe que devrait se faire la suffixation en -er en français, et non pas à partir de twit - comme c'était le cas dans cette entrée. Il en résulte que le nom propre en anglais fonctionne comme verbe en français.

A part cela nous trouvons dans $\mathrm{La}$ Parlure d'autres cas de dérivation, dont nous citerons un exemple :

\section{Sarkozyr ${ }_{\text {(France) }}$}

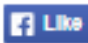

A Ajouter une définition et un exemple

J'ai déjà entendu ça au/en... Choisir $v$

Définition: revoir à la baisse

4 pour, 0 contre

Exemple: J'ai dû sarkozyr mes prétentions.

Ajouté le 04 jui 2011.

Définition: Diminuer

Exemple: $\mathrm{Ca}$ fait 3 jours que jai pas dormi, ma capacité à réfléchir est toute

1 pour, 0 contre

sarkozye.

Ajouté le 04 jui 2011.

Définition: Réduire (verbe)

2 pour, 0 contre

Exemple: Avec la bulle immobilière, la surface des appartements à Paris ne fait que

sarkozyr d’année en année.

Ajouté le 04 jui 2011

Définition: Rapetisser, rétrécir (verbe)

Exemple: Depuis quelques années, mon salaire sarkozyt de plus en plus.

Ajouté le 04 jui 2011.

Le nom propre à la base de ce verbe n'est explicité ni dans les gloses ni dans les exemples, mais il est facilement reconnaissable. La graphie du suffixe verbal $-y r$, atypique pour l'orthographe française, enlève toute ambiguïté.

Que peut-on retenir du nom propre par rapport à l'innovation lexicale? Il faut distinguer deux cas différents. D'une part, les noms propres forment de nombreuses locutions imagées, et leur emploi reste alors majoritairement métaphorique. D'autre part, le nom propre peut subir des modifications 
morphosyntaxiques (sarkozyr) et/ou graphiques (Môsusse). Ces modifications se font à partir des matrices internes ou externes (Tweeter). Dans tous ces cas, la nature aussi bien que la signification des noms propres changent radicalement.

\section{Conclusion}

Nous avons traité dans cet article des dictionnaires collaboratifs, de leur structure, de leur forme et de leur fonctionnement. En comparaison des dictionnaires classiques, ils présentent plusieurs particularités notables ; soulignons avant tout que la création des entrées ainsi que la rédaction des gloses définitoires et des exemples d'usage est laissée aux internautes. Les rédacteurs sont donc multiples (d'où la multiplicité des formes des articles) et, d'autre part, il s'agit en grande partie de non-spécialistes. Les dictionnaires collaboratifs comportent des manifestations de la langue orale et des usages discursifs nouveaux. De ce fait, les articles sont riches en néologismes et formes émergentes car «l'état néologique est mouvant par excellence » (Candel 2003 : 245). Nous nous sommes intéressés plus particulièrement au dictionnaire $L a$ Parlure, qui est un dictionnaire collaboratif français, donc intéressant sous le double rapport de sa dimension francophone et de son fonctionnement interactif.

Dans un deuxième temps, nous nous sommes posé la question du nom propre, traité là tout autrement que par la lexicographie traditionnelle. A la différence des dictionnaires classiques, il est inséré directement dans la nomenclature et son traitement reste forcément moins systématique, ce qui s'explique par la nature même de ces dictionnaires. En ce qui concerne La Parlure, le nom propre y figure dans une mesure importante, sous un aspect soit inchangé soit modifié. Dans le premier cas, il s'agit des entrées où le nom propre se présente directement tel quel, c'est-à-dire non-modifié du point de vue morphosyntaxique. Nous y trouvons aussi bien des noms propres standard que des noms métaphoriques. Ces derniers se caractérisent par le changement de référent via la création d'un lien métaphorique. Dans La Parlure, les noms métaphoriques sont largement plus présents que les noms propres stricto sensu (une trentaine contre une quinzaine), contrairement aux dictionnaires classiques où ce type d'expressions est absent.

Dans le second cas, on pourrait estimer que le nom propre fait partie d'un processus relevant de la création lexicale. D'une part, il peut subir diverses modifications morphosyntaxiques (une quinzaine d'occurrences dans le corpus): il s'agit le plus souvent de siglaison, conversion ou suffixation. Le contenu de ces entrées reste très varié, leur but étant de créer de nouvelles unités lexicales dites aussi néologismes formels (Sablayrolles 2003 : 10). D'autre part, les noms propres peuvent faire partie de locutions, ce qui est le cas dominant (une centaine d'occurrences dans La Parlure, où ces locutions figurent en totalité dans les entrées, au contraire des dictionnaires classiques, où les syntagmes n'apparaissent ni en entrées ni en sous-entrées). Cela démontre que le nombre de locutions se basant sur le nom propre est plus important que le nombre d'entrées provenant directement d'un nom propre. Cette catégorie est donc de loin la plus importante, avec un nombre élevé d'anthroponymes, suivi de toponymes (sur ce point, La Parlure rejoint les dictionnaires classiques). La valeur des noms propres dans ces locutions est principalement métaphorique, leur interprétation dépendant étroitement du contexte énonciatif.

On retiendra en somme que dans La Parlure le nom propre se trouve au sein de divers types d'entrées, soit dans les noms propres à proprement parler, soit dans les noms et locutions métaphoriques soit dans les néologismes créés à partir de noms propres. En conclusion, nous pouvons dire que non seulement la place du nom propre dans les dictionnaires collaboratifs est significative, mais également que les noms propres se trouvent au cœur de l'innovation linguistique actuelle en langue française. 


\section{Remerciements}

Nous remercions Greg Sadetsky, l'éditeur du dictionnaire La Parlure, pour tout le matériel, les informations et l'intérêt qu'il porte pour le projet de cet article.

\section{Références bibliographiques}

Candel, D. (2003). Quelle néologie pour un grand dictionnaire de langue ? In : Sablayrolles (2003), pages 247-260.

Cormier, A. (2011). Rôle de l'énonciation dans l'analyse linguistique du nom propre. Thèse de doctorat.

Cormier, A. (2013). Rôle de l'énonciation dans l'analyse linguistique du nom propre. Limoges : Lambert Lucas.

Eluerd, R. (2000). La lexicologie. Que sais-je ? Paris : PUF.

Gadet, F. (2007). La variation sociale en français. Paris : Ophrys.

Gary-Prieur, M. N. (1994). Grammaire du nom propre. Paris : PUF.

Gardiner, A. H. (1954). The Theory of Proper Names : A Controversial Essay. Londres : Oxford University Press.

Goffman, E. (1987). Façons de parler. Paris : Minuit. (Forms of Talk, 1981).

http://correcteurs.blog.lemonde.fr/2010/05/20/de-quoi-sarkozyr-est-il-le-nom/ [consulté en mars 2014].

Honvault, R. (2003). Innovation lexicale et orthographe. In : Sablayrolles (2003), pages 37-57.

Jonasson, K. (1991). Les noms propres métaphoriques : construction et interprétation. Langue française $\mathrm{n}^{\circ} 92$, pages 64-82.

Jonasson, K. (1994). Le nom propre. Constructions et interprétations. Louvain-La-Neuve : Duculot.

Kleiber, G. (1981). Problèmes de référence: descriptions définies et noms propres. Metz: Centre d'Analyse Syntaxique.

Le Grand Larousse illustré (2014). Paris : Larousse.

Le Petit Robert (2014). Paris : Le Robert.

Leroy, S. (2004). Le nom propre en français. Paris : Ophrys.

Leroy, S. (2001). Entre identification et catégorisation, l'antonomase du nom propre en français. Thèse de doctorat.

Niklas-Salminen, A. (1997). La lexicologie. Paris : Armand Colin.

Mérillou, C. (2003). Innovation lexicale et interférence de langues : Le cas du français québécois. In : Sablayrolles (2003), pages 389-408.

Mufwene, S. (1988). Dictionaries and proper names. International Journal of Lexicography 1/3, pages 268-283.

Pruvost, J. et J.-F. Sablayrolles. (2003). Les néologismes. Que sais-je ? Paris : PUF.

Quemada, B. (1967). Les dictionnaires du français moderne, 1539-1863 : Étude sur leur histoire, leurs types et leurs méthodes. Paris : Didier.

Rey, A. (2008). De l'artisanat des dictionnaires à une science du mot. Images et modèles. Paris : Armand Colin.

Sablayrolles, J.-F. (2000). La néologie en français contemporain. Examen du concept et analyse de productions néologiques récurrentes. Paris : Honoré Champion.

Sablayrolles, J.-F. (éd.). (2003). L'innovation lexicale. Paris : Honoré Champion.

Thauvin-Chapot, A. (2003). Innovation lexicale et français langue étrangère ou langue seconde. In : Sablayrolles (2003), pages 429-436. 
Schnedecker, C. (1997). Nom propre et chaines de référence. Recherches linguistiques, $n^{\circ} 21$.

Vaxelaire, J.-L. (2005). Les noms propres. Une analyse lexicologique et historique. Paris : Honoré Champion.

www.dictionnairedelazone.fr [consulté en mars 2014]

www.laparlure.com [consulté en mars 2014]

www.neologisms.us [consulté en mars 2014]

www.ostarrichi.org [consulté en mars 2014]

www.razvezanijezik.org [consulté en mars 2014]

www.urbandictionary.com [consulté en mars 2014]

www.wordnik.com [consulté en mars 2014] ${ }^{1}$ Pour des raisons pratiques, nous nous permettons de citer les entrées dans le texte sous la forme suivante : le mot-
vedette, la glose définitoire et l'exemple d'usage sont séparés par les tirets et le tout est en italique. Nous présentons
les entrées telles qu'elles figurent dans le dictionnaire, en respectant les (dys)graphies originelles.

${ }^{2}$ Nous ne tenons pas compte ici des noms propres dans les locutions figurant dans les entrées. 